\title{
Prognostic Significance of Cyclin D1 Over-expression in Colorectal Cancer: An Experience from Madinah, Saudi Arabia
}

\author{
Abdulkader Mohammed Albasri ${ }^{1 *}$, Mohammed Aboulmatty Elkablawy ${ }^{1,2}$, Irfan \\ Altaf Ansari ${ }^{1}$, Ahmed Safar Alhujaily ${ }^{3}$
}

\begin{abstract}
Background and study aim: Cyclin D1 is a key regulatory protein in the cell cycle and is over-expressed in many tumors, including endometrial, thyroid, urothelial, breast, brain gliomas, and esophageal cancers. The main aim of the present study is to examine the expression pattern of cyclin D1 and its correlation with the different clinicopathological features in patients with colorectal camcer (CRC) from the Madinah region of Saudi Arabia. Patients and methods: The archival tumor blocks were analyzed using immunohistochemistry for Cyclin D1 over-expression in 324 CRC patients diagnosed from January 2006 to December 2017, at the Department of Pathology, King Fahad Hospital, Madinah, Saudi Arabia. Results: Cyclin D1 over-expression was absent in normal mucosa, while $15 \%$ cases of adenoma showed its over-expression. In CRC, Cyclin D1 was expressed at high levels in $24.1 \%$ of case. No significant correlation was observed between Cyclin D1 over-expression and age, gender, tumor size, type and location. However, Cyclin D1 over-expression exhibited a significant correlation with tumor differentiation $(\mathrm{p}=0.04)$, lymph node involvement $(\mathrm{p}=0.001)$, lymphovascular invasion $(\mathrm{p}=0.001)$, distant metastasis $(\mathrm{p}=0.006)$ and AJCC staging $(\mathrm{p}=0.001)$. The Kaplan-Meir analysis revealed a shorter period of survival with Cyclin D1 over-expression $(\mathrm{p}=0.000)$. The Cox-regression model analysis showed that Cyclin D1 over-expression was an independent prognostic marker in CRC ( $\mathrm{p}=0.000)$. Conclusion: Cyclin D1 over-expression increases during normal-adenoma-carcinoma sequence. The significant association observed between Cyclin D1 over-expression, advanced tumor stage and short survival period clearly suggest the role of Cyclin DI in the carcinogenesis and progression of CRC.
\end{abstract}

Keywords: Cyclin D1- colorectal- cancer- prognosis

Asian Pac J Cancer Prev, 20 (8), 2471-2476

\section{Introduction}

Colorectal cancer (CRC) is one of the most common cancers, being the second most common cancer in females and the third in males. Colorectal cancer statistics in 2013 showed an incidence of nearly 14.1 million cases and 694,000 deaths around the globe (Alao, 2007). The majority of cases of CRC occur in the developed nations, the incidence being lower in Asian and African countries. The Kingdom of Saudi Arabia (KSA) is an area of low incidence; however, according to the latest Saudi cancer registry, $\mathrm{CRC}$ is the second most common cancer, and the trend, disturbingly, is upward (Albasri et al., 2014; Al-Maghrabi et al., 2015). In spite of a better diagnostic approach and multimodal therapeutic regimens, CRC is nevertheless associated with a poor outcome, and approximately $50 \%$ to $60 \%$ of patients die within five years of diagnosis (Arber et al., 1996). Moreover, in KSA, a recent histopathology-based study from the Madinah region by Albasri et al., (2014) reported that CRC was diagnosed at an advanced stage in their cohort.

The multi-step genetic alteration in CRC is well studied: a series of events leads to an alteration of the normal epithelium, then to precancerous lesionsand further to adenocarcinoma, and subsequently to the development of metastasis (Bahnassy et al., 2004). Abnormal regulation of cell proliferation is the central event in tumor progression, generally reflected by alteration in the cell cycle. Therefore, a better understanding of cell cycle regulation is important for interpreting the significance of alterations. During the cell cycle process, a cell is committed to DNA replication, and the cycle starts with the Gap 1 (G1) phase (Bahnassy et al., 2004; Balcerczak et al., 2005). The checkpoint in the G1phase is known as the restriction point, beyond which the cell becomes independent and no growth factors are required for entering the synthesis phase (S phase); the cell is then committed to complete the cycle. These checkpoints are carefully controlled by

${ }^{1}$ Department of Pathology, Taibah University, Universities Road, ${ }^{3}$ Department of Pathology, King Fahd Hospital, Al-Madinah Al-Munawwarah, Kingdom of Saudi Arabia, '2Department of Pathology, Menofia University, Menofia, Egypt. *For Correspondence: abdbasri@hotmail.com 
cyclins, especially cyclin D1 (Bali et al., 2004; Chen et al., 2007). Cyclin D1, a key regulatory protein in the cell cycle, is encoded by the CCND1 gene, located on chromosome 11q13, and over-expression of this protein interrupts normal cell cycle control, thus encouraging the development and progression of cancer (Cunningham et al., 2010). Cyclin D1 over-expression has been observed in many tumors, including endometrial, thyroid, urothelial, breast, brain gliomas, and esophageal cancers (Elkablawy and Albasri, 2015). The up-regulation of cyclin D1 plays an important role in the pathogenesis and metastases of $\mathrm{CRC}$, and seems to be a useful prognostic marker for CRC (Bali et al., 2004; Chen et al., 2007). The main objective of the present study is to examine the expression pattern of cyclin D1 and its correlation with the different clinicopathological features in patients from the Madinah region of Saudi Arabia.

\section{Materials and Methods}

The present study was a retrospective study involving archival paraffin blocks of tumor from 324 consecutive patients with CRC diagnosed between January 2006 and December 2017 in the histopathology laboratory of King Fahad Hospital (a tertiary care hospital) in the Madinah region of Saudi Arabia. The clinicopathological data included sex, age, tumor site, size, type, grade, lymph node status, lymphovascular invasion, distant metastasis status, and the American Joint Committee on Cancer (AJCC) stage, all of which were obtained from patients' medical records. The present study did not involve patients' personal information or have any implicationsfor the management plan. Hence, no ethical approval was sought, according to the principles of the Helsinki Declaration. Twenty cases of normal colonic mucosa and 40 cases of colorectal adenomas were used as controls in the present study. Only histopathologically confirmed invasive CRC cases who underwent total colectomies, hemi-colectomies, and local resection were included. In-situ lesions, recurrences, biopsies, benign lesions, and metastatic lesions were excluded from the analysis.

\section{Immunohistochemical procedures}

Tissue cores were extracted from archival blocks of the primary CRC cases and used in the construction of a tissue mini-array (TmA), asdescribed previously by Elkablawy and Albasri (Ferlay et al., 2013). Four micro-meter-thick tissue sections were cut from the TmA blocks, mounted on positively charged poly-1-lysil slides, and immunohistochemistry was performed on them using the avidin-biotin detection system, following the instructions of the manufacturer.The human anti-cyclin D1 monoclonal antibody was used (diluted 1:70 in blocking solution; Ventana, Inc., Tucson, AZ, USA). The immunohistochemistry procedure was done on an automated immune-stainer (Ventana Bench Mark $\mathrm{Xt}$; Ventana, Inc.). The positive control was achieved by using a case of breast cancer, and the negative control was achieved by substitution of the primary antibody with serum.
Interpretation of immunohistochemical staining

The percentage of nuclear staining of cyclin D1 was reported as: 0 , less than $5 \% ; 1,5-25 \% ; 2,26-50 \% ; 3$, $51-75 \%$; and 4 , more than $75 \%$. The staining pattern was scaled from 0 to 3 , where 0 was negative, 1 was weak, 2 was moderate, and 3 was strong. The final expression score was calculated as follows: '-' for score 0 , '+' for scores $1-3$, ' ++ ' for scores 4-6, and ' +++ ' for scores $>6$. For the purpose of statistical analysis, the cases that scored '-' and '+'as a low score were comparedwith the cases that scored ' ++ ' and ' +++ ' as a high score. No cytoplasmic staining of cyclin D1 was found in any of the studied cases.

\section{Statistical analysis}

Data analysis was performed using the Statistical Package for Social Sciences (SPSS) Version 22.0 for Windows XP (BMCorp., Armonk, NY, USA). To determine inter-observer reproducibility,correlation analysis of the two data sets was performed. The chi-square test and Fisher's exact tests were performed to extract the significant level of association between cyclin DI over-expression and the clinicopathological parameters. The cumulative survival analysis was assessed by the univariate Kaplan-Meir method, and the long-rank test was performed for comparison of the survival curves. Risk factor analysis of factors independently associated with survival was carried out by the Cox proportional hazards linear regression model. A p value of $<0.05$ was considered as significant for all the statistical analyses. The present study was a retrospective study involving the archival histopathology material and record; and did not involve patients' personal information or any implication upon the management; thus, was according to the principles of Helsinki Declaration. Hence no ethical approval was mandatory in the present study.

\section{Results}

\section{Clinicopathological characteristics of the cases}

In the present study, a total of 324 cases of CRC was included. Therewere 196 male patients $(60.5 \%)$ and 128 female patients $(39.5 \%)$, making a male to female ratio of 1.5:1.The ages of the studied cases ranged from 22 to 96 years, with a mean age of 56.9 years. Leftand right-sided tumors were seen in $63.5 \%$ and $36.5 \%$ of cases, respectively, and the size of the tumor was $>4 \mathrm{~cm}$ in $84 \%$ of cases. The commonest histological type was adenocarcinoma, seen in 279 cases $(86.1 \%)$, and the majority of the tumors were moderately differentiated. AJCC stages I, II, III, and IV were reported in $10.5 \%, 44.7 \%, 31.5 \%$, and $13.3 \%$ of cases, respectively. Approximately $55.2 \%$ of cases had positive lymph node metastasis and lymphovascular invasion. Distant metastases were seen in $30.3 \%$ of cases. Table 1 summarizes the clinicopathological characteristics of the 324 CRC cases.

\section{Cyclin D1 over-expression profiles}

Evaluation of cyclin D1 protein over-expression on TmA sections containing normal colonic mucosa showed a complete absence of its expression. By contrast, cyclin 
Table 1. The Clinicopathological Features of CRC Cases

\begin{tabular}{|c|c|c|}
\hline Variable & No of Patients & $\%$ of Total \\
\hline \multicolumn{3}{|l|}{ Age (yr) } \\
\hline$<40$ & 177 & 54.6 \\
\hline$\geq 40$ & 147 & 45.4 \\
\hline \multicolumn{3}{|l|}{ Sex } \\
\hline Female & 128 & 39.5 \\
\hline Male & 196 & 60.5 \\
\hline \multicolumn{3}{|l|}{ Tumor site } \\
\hline Colon & 168 & 51.9 \\
\hline Rectum & 156 & 48.1 \\
\hline \multicolumn{3}{|l|}{ Tumor size } \\
\hline$<4 \mathrm{~cm}$ & 52 & 16 \\
\hline$\geq 4 \mathrm{~cm}$ & 272 & 84 \\
\hline \multicolumn{3}{|l|}{ Tumor type } \\
\hline Adenocarcinoma & 279 & 86.1 \\
\hline Mucinous adenocarcinoma & 45 & 13.9 \\
\hline \multicolumn{3}{|l|}{ Tumor differentiation } \\
\hline Well & 49 & 15.1 \\
\hline Moderate & 260 & 80.2 \\
\hline Poor & 15 & 4.7 \\
\hline \multicolumn{3}{|l|}{ Lymph Node } \\
\hline Negative & 179 & 55.2 \\
\hline Positive & 145 & 44.8 \\
\hline \multicolumn{3}{|l|}{ Lymphovascular invasion } \\
\hline Negative & 179 & 55.2 \\
\hline Positive & 145 & 44.8 \\
\hline \multicolumn{3}{|l|}{ Distant metastasis } \\
\hline Negative & 281 & 86.7 \\
\hline Positive & 43 & 13.3 \\
\hline \multicolumn{3}{|l|}{ AJCC } \\
\hline I & 34 & 10.5 \\
\hline II & 145 & 44.7 \\
\hline III & 102 & 31.5 \\
\hline IV & 43 & 13.3 \\
\hline
\end{tabular}

D1 over-expression was noted in six out of 40 cases (15\%) ofcolorectal adenoma. In CRC cases, cyclin D1 was expressed at a low level in $246(75.9 \%)$ cases, while 78 (24.1\%) cases showed high levels ofexpression (Figure 1).

Correlation of cyclin D1 over-expression with the clinicopathological parameters

Cyclin D1 over-expression did not reveal any significant correlation with age, gender, tumor size, tumor histologic type,ortumor location. However, cyclin D1 over-expression exhibited a significant correlation with tumor differentiation $(\mathrm{p}=0.04)$, lymph node involvement $(p=0.001)$, lymphovascular invasion $(p=0.001)$, distant metastasis $(p=0.006)$, and AJCC staging $(p=0.001)$. A summary of our observations of correlation of cyclin D1 over-expression with clinicopathological parameters ispresented in Table 2 .

Univariate and multivariate long-term survival analysis The significant findings $(\mathrm{p}<0.05)$ associated with survival for all the 324 CRC cases in the present study are shown in Table 3 . The significant survival curves for cyclin D1 over-expression are shown in Figure 2. The patients with a high score forcyclin D1 over-expression experienced a significantly lower survival period than the patients with a low score of cyclin D1 over-expression $(\mathrm{p}=0.000)$. High cyclin D1 expression $(\mathrm{p}=0.000)$, AJCC stage $(p<0.001)$, lymph node metastasis $(p<0.001)$, lymphovascular invasion $(\mathrm{p}<0.001)$, distant metastasis $(p<0.001)$, and histological tumor grade $(p<0.001)$ were the only significant independent prognostic indicators, as determined by multivariate analysis usingthe Cox regression model.

\section{Discussion}

The incidence of CRC has reportedly increased in nations where it was historicallyless prevalent (Asaad et al., 2000; Hilska et al., 2005). In the past decades, advances in molecular carcinogenesis have described

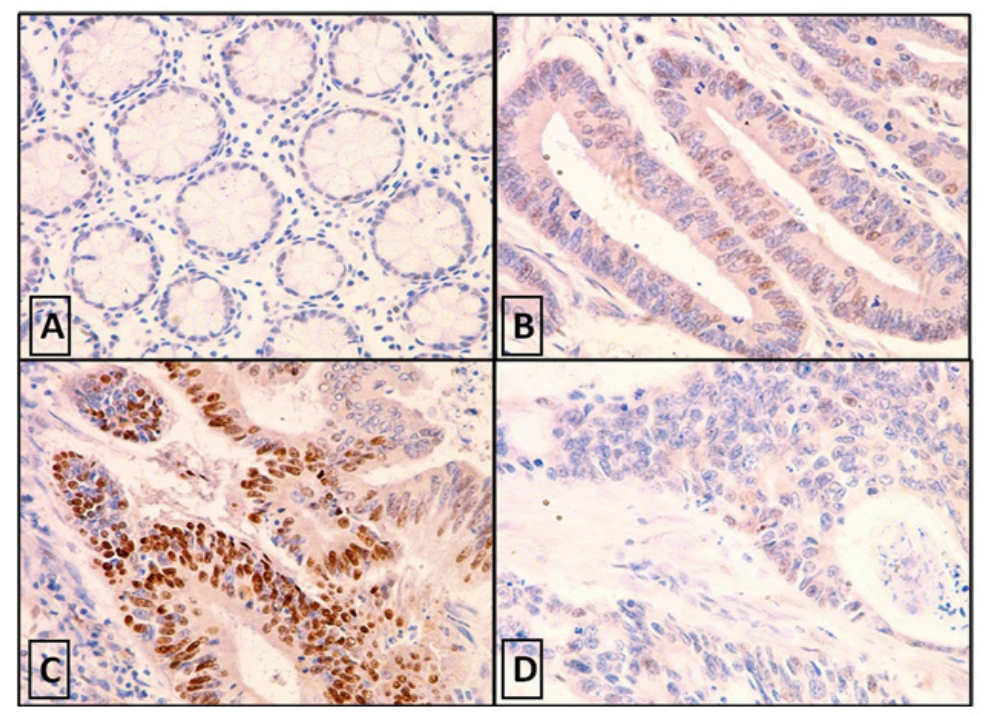

Figure 1. Different Patterns of Immunohistochemical Cylin D1 Expression in A. Normal colon (no staining), B. Colonic adenoma (mild to moderate nuclear positivity), C. Colorectal carcinoma (marked nuclear expression), D. Colorectal carcinoma (low nuclear expression). 
Table 2. Correlation of Cylin D1 Over-expression with the Clinicopathological Variables

\begin{tabular}{|c|c|c|c|}
\hline Variable & $\begin{array}{c}\text { Low Cycline } \\
\text { D1 }\end{array}$ & $\begin{array}{c}\text { High Cycline } \\
\text { D1 }\end{array}$ & $P$ value \\
\hline Age (yr) & & & $=0.091$ \\
\hline$<40$ & 141 & 36 & \\
\hline$\geq 40$ & 105 & 42 & \\
\hline Sex & & & $=0.59$ \\
\hline Male & 151 & 45 & \\
\hline Female & 95 & 33 & \\
\hline Tumor site & & & $=0.897$ \\
\hline Colon & 127 & 41 & \\
\hline Rectum & 119 & 37 & \\
\hline Tumor size & & & $=0.72$ \\
\hline$<4 \mathrm{~cm}$ & 41 & 11 & \\
\hline$\geq 4 \mathrm{~cm}$ & 205 & 67 & \\
\hline Tumor type & & & $=0.70$ \\
\hline Adenocarcinoma & 213 & 66 & \\
\hline Mucinous adenocarcinoma & 33 & 12 & \\
\hline Tumor differentiation & & & $=0.04$ \\
\hline Well & 43 & 6 & \\
\hline Moderate & 194 & 66 & \\
\hline Poor & 9 & 6 & \\
\hline Lymph Node & & & $=0.001$ \\
\hline Negative & 149 & 30 & \\
\hline Positive & 97 & 48 & \\
\hline Lymphovascular invasion & & & $=0.001$ \\
\hline Negative & 149 & 30 & \\
\hline Positive & 97 & 48 & \\
\hline Distant metastasis & & & $=0.006$ \\
\hline Negative & 221 & 60 & \\
\hline Positive & 25 & 18 & \\
\hline AJCC stage & & & $=0.001$ \\
\hline I & 31 & 3 & \\
\hline II & 118 & 27 & \\
\hline III & 72 & 30 & \\
\hline IV & 25 & 18 & \\
\hline
\end{tabular}

various crucial signaling pathways of the cell cycle in the development and progression of CRC. One such pathway involves the over-expression of cyclin D1. Cyclin D1 is an important checkpoint regulator protein at the G1 to S phase transition (Bali et al., 2004; Chen et al., 2007). Cyclin D1 can act as an oncogene during tumorigenesis by modulating certain processes such as excessive growth, invasion, angiogenesis, and resistance to apoptosis (Holland et al., 2001). The carcinogenic role of cyclin D1 over-expression has been reported in the cancers of various organs, includingCRC, and this over-expression appears to be associated with aggressive behavior and worse prognosis (Elkablawy and Albasri, 2015 ).

Recently, an immunohistochemistry-based meta-analytical study of cyclin $D 1$ expression in squamous cell carcinoma of the esophagus revealed a significant association between cyclin D1 expression and aggressive tumor histology, lymph node metastasis, and higher AJCC stages (Hur et al., 2000). Asimilar study on estrogen-positive (ER) breast cancers showed that cyclin D1 over-expression was associated with the worst clinicopathological features and prognosis (Ibrahim et al., 2008). Moreover, cyclin D1 over-expression is also reportedly associated with poor survival outcomes in patients with serous ovarian cancers and non-small-cell carcinoma of the lung (International Association of Cancer Registries, 2017; Jang et al., 2012).

The role of cyclin D1 over-expression in CRC is somewhat controversial. While some reports have observed a significant association between its expression and poor survival outcomes, others have reported it as a marker of a good prognosis (Jares et al., 1994; Li et al., 2014), and still others have reported no significant association between cyclin D1 expression and prognosis (Mate et al., 1996; Maeda et al., 1997). In view of these controversial reports, we evaluated the cyclin Dlover-expression pattern by immunohistochemistry on TmA blocks in CRC cases. Three patterns of cyclin D1 over-expression by immunohistochemistry were demonstrated in previous studies, i.e., nuclear, cytoplasmic, and nuclear plus cytoplasmic. However, for CRC, only the nuclear staining pattern was considered to be significant (McKay et al.,

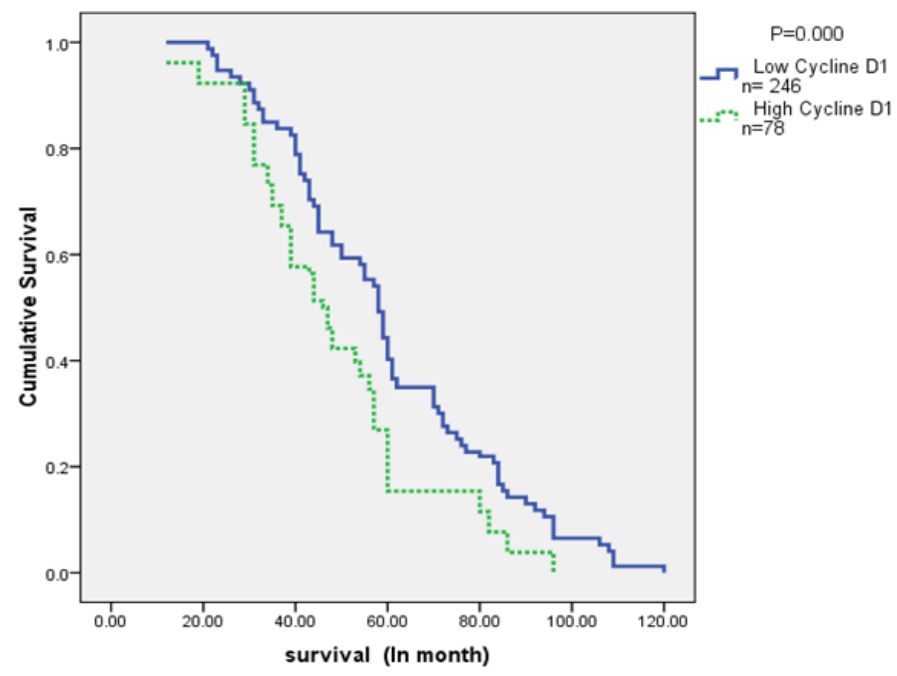

Figure 2. Shows Kaplan-Meier Curves for Significant Overall Survival Functions for Cylin D1 Expression in CRC 
Table 3. Test Statistics for Equality of Survival Distribution for Prognostic Factors Examined in 324 Colorectal Carcinomas. A Univariate Approach to Cancer-specific Mortality

\begin{tabular}{lccc}
\hline Factor & $\begin{array}{c}\text { Univariate } \mathrm{X}^{2} \text { for } \\
\text { the log-rank }\end{array}$ & Df. & P value \\
\hline Clinicopathological factors: & 772.81 & 3 & $<0.001$ \\
AJCC stage & 400.28 & 1 & $<0.001$ \\
Lymph node stage & 507.58 & 1 & $<0.001$ \\
Metastasis stage & 400.28 & 1 & $<0.001$ \\
Lymphovascular invasion & 170.27 & 2 & $<0.001$ \\
Histological tumour grade & 2.141 & 1 & 0.133 \\
Tumour size & 0.366 & 1 & 0.545 \\
Tumour site & 1.153 & 1 & 0.283 \\
Tumour type & 0.308 & 1 & 0.579 \\
Age & 0.064 & 1 & 0.800 \\
Sex & & & \\
Immunostaining & 15.96 & 1 & $=0.000$ \\
Cycline D1 immunostaining & & & \\
\hline
\end{tabular}

2002; Li et al., 2014).

In our study, evaluation of cyclin D1 over-expression on TmA sections containing normal mucosa, colorectal adenomas, and colorectal carcinomas revealed a negative expression pattern in normal colonic mucosa and a gradual increase in its detection in the normal to adenoma to carcinoma sequence. This suggested an oncogenic role of cyclin Dl over-expression in colorectal carcinogenesis. Arber et al., (1996) reported cyclin Dl over-expression in $30 \%$ of adenomas, and concluded this phenomenon was possibly an early event during tumorigenesis. This sequential up-regulation of cyclin Dl over-expression from normal to adenoma to carcinoma in our study is in accordance with the findings of previous authors, indicating an oncogenic role of cyclin D1 in colorectal carcinogenesis (Bahnassy et al., 2004; Jiang et al., 2006; Li et al., 2014).

In contrast to normal mucosa and adenoma, cyclin D1 over-expression was observed as a low score in $75.9 \%$ of cases and a high score in $24.1 \%$ of cases. Several previous studies on CRC reported cyclin D1 positivity in a range as low as $23.1 \%$ to as high as $100 \%$ (Myklebust et al., 2012; Ogino et al., 2009). This wide range of expression may be attributed largely to factors such as cohort size, type of antibody used, antigen retrieval techniques, and the scoring system. In the present study, the level of expression was more intense or high in the poorly differentiated carcinomas. The correlation between high histopathological grade and cyclin Dl over-expression has also been reported in other tumors, such as non-small-cell lung carcinomas (Pasz-Walczak et al., 2001), squamous cell carcinomas of the larynx (Sterlacci et al., 2010), squamous cell carcinomas of the esophagus (Hur et al., 2000), and breast carcinomas (Ibrahim et al., 2008).

In our study, no significant correlation was observed between the cyclin D1 over-expression and patients' gender, tumor size, tumor histologic type, and tumor location.Similar findings of no significant correlation with these clinicopathological parameters were reported by various previous authors (Bahnassy et al., 2004; Jiang et al., 2006; Li et al., 2014). However, a significant correlation was seen with the patient's age, lymph node metastasis, lymphovascular invasion, distant metastasis, and AJCC stage. Previous studies by Assaad et al., (2000), Belcerczak et al., (2005), and Almaghrabi et al., (2015) from different parts of the world found a similar strong correlation between pathological tumor stage and cyclin D1 over-expression. According to Belcerczak et al., (2005) and Almaghrabi et al., (2015), among all clinicopathological parameters, lymph node metastasis and lymphovascular invasion showed the strongest correlation with cyclin Dl over-expression, as observed also in our study. Hence, our observations of a significant association betweencyclin D1 over-expression and different clinicopathological parameters are in agreement with previous studies from the region and around the world.

In univariate and multivariate analysis, contrasting results have been documented on the prognostic and predictive value of cyclin D1 over-expression. Our findings in the present study support that high cyclin D1 over-expression is associated with a shorter survival time and poor patient outcomes, as compared to low levels of cyclin D1 over-expression. Several previous studies have yielded a controversial result relating to the prognostic significance of cyclin Dl over-expression in CRC patients. Similar to our findings of a significant association, Bahnassy et al., (2004), Mykelbust et al., (2012), Ogino et al., (2009), and Li et al., (2014) reported cyclin D1 over-expression as a poor prognostic indicator and associated with a short survival period. By contrast, McKay et al., (2002) observed that cyclin D1 expression in $\mathrm{CRC}$ is associated with a survival advantage for patients. However, Assaad et al., (2000), Von Stockmar-Von Wangenheim et al., (2008), and Almaghrabi et al., (2015) found no association between cyclin D1 expression and patients' survival. In the present study, cyclin D1 turned out to be an independent prognostic marker for CRC, similar to the findings of Bahnassy et al., (2004).

In conclusion, our findings of an increasing percentage of cyclin D1 over-expression in the sequence from normal to adenoma to carcinoma suggest an oncogenic role for cyclin D1 in CRC patients. We observed a significant correlation with lymph node metastasis, lymphovascular invasion, distant metastasis, and advanced AJCC stages. Moreover, a significant short survival period was noted in patients with high cyclin D1 over-expression. Lymph node metastasis, lymphovascular invasion, distant metastasis, and advanced AJCC stages were the independent prognostic indicators, as calculated by multivariate analysis usingthe Cox regression model. Our findings confirmed the important role of cyclin D1 in CRC carcinogenesis and can be used as a reliable prognostic indicator in CRC patients.

\section{Acknowledgements}

The authors thank the Deanship of Scientific Research, Taibah University, Almadinah Almonawwarah, Saudi Arabia, for their encouragement and support. The authors 
thank Dr. Alla Khadri (pathology Department, Taibah University, KSA) for revising the grammer and language. The authors thank Mrs. Faten Bokhary (Lab. technician, Taibah University, KSA) for technical advice.

\section{References}

Alao JP (2007). The regulation of cyclin D1 degradation: roles in cancer development and the potential for therapeutic invention. Mol Cancer, 6, 24.

Albasri A, Yosef H, Hussainy AS, et al (2014). Histopathological features of colorectal cancer in Al-Madinah region of Saudi Arabia. Asian Pac J Cancer Prev, 15, 3133-7.

Al-Maghrabi J, Mufti S, Gomaa W, et al (2015). Immunoexpression of cyclin D1 in colorectal carcinomas is not correlated with survival outcome. J Microsc Ultrastruct, 3, 62-7.

Arber N, Hibshoosh H, Moss SF, et al (1996). Increased expression of cyclin D1 is an early event in multistage colorectal carcinogenesis. Gastroenterology, 110, 669-74.

Asaad NY, Kandil MA, Mokhtar NM (2000). Prognostic value of Cyclin D1 and $p 53$ protein in colorectal carcinoma. J Egypt Nat Cancer Inst, 12, 283-92.

Bahnassy AA, Zekri AR, El-Houssini S, et al (2004). Cyclin A and cyclin D1 as significant prognostic markers in colorectal cancer patients. BMC Gastroenterol, 4, 22.

Balcerczak E, Pasz-Walczak G, Kumor P, et al (2005). Cyclin $D 1$ protein and $C C N D 1$ gene expression in colorectal cancer. Eur J Surg Oncol, 31, 721-6.

Bali A, O'Brien PM, Edwards LS, et al (2004). Cyclin D1, p53, and $p 21 \mathrm{Wafl} / \mathrm{Cip} 1$ expression is predictive of poor clinical outcome in serous epithelial ovarian cancer. Clin Cancer Res, 10, 5168-77.

Chen WC, Lin MS, Zhang BF, et al (2007). Survey of molecular profiling during human colon cancer development and progression by immunohistochemical staining on tissue microarray. World J Gastroenterol, 13, 699-708.

Cunningham D, Atkin W, Lenz HJ, et al (2010). Colorectal cancer. Lancet, 375, 1030-47.

Elkablawy MA, Albasri AM (2015). High quality tissue miniarray technique using a conventional $\mathrm{TV} /$ radio telescopic antenna. Asian Pac J Cancer Prev, 16, 1129-33.

Ferlay J, Soerjomataram I, Dikshit R, et al (2013). Globocan 2012 v1.0, Cancer Incidence and Mortality Worldwide: IARC Cancer Base No. 11 (Internet) \{2015 November 2015). Lyon, France: International Agency for Research on Cancer; Available from: http://globocan.iarc.fr.

Hilska M, Collan YU, O Laine VJ, et al (2005). The significance of tumor markers for proliferation and apoptosis in predicting survival in colorectal cancer. Dis Colon Rectum, 48, 2197-208.

Holland TA, Elder J, McCloud JM, et al (2001). Subcellular localisation of cyclin D1 protein in colorectal tumours is associated with $\mathrm{p} 21 \mathrm{WAF} 1 / \mathrm{CIP} 1$ expression and correlates with patient survival. Int $J$ Cancer, 95, 302-6.

Hur K, Kim JR, Yoon BI, et al (2000). Overexpression of cyclin $D 1$ and cyclin $\mathrm{E}$ in 1, 2-dimethylhydrazine dihydrochlorideinduced rat colon carcinogenesis. J Vet Sci, 1, 121-6.

Ibrahim EM, Zeeneldin AA, El-Khodary TR, et al (2008). Past, present and future of colorectal cancer inthe Kingdom of Saudi Arabia. Saudi J Gastroenterol, 14, 178-82.

International Association of Cancer Registries (2017). Saudi Cancer Incidence Report 2014. Saudi Cancer Registry. Riyadh (KSA):King Faisal Specialist Hospital and Research Center Council of Health Services.

Jang KY, Kim YN, Bae JS, et al (2012). Expression of cyclin D1 is associated with b-catenin expression and correlates with good prognosis in colorectal adenocarcinoma. Transl
Oncol, 5, 370-8.

Jares P, Fernandez P, Campo E, et al (1994). PRAD-1/cyclin D1 gene amplification correlates with messenger RNA overexpression and tumor progression in human laryngeal carcinomas. Cancer Res, 54, 4813-7.

Jiang J, Wang J, Suzuki S, et al (2006). Elevated risk of colorectal cancer associated with the AA genotype of the cyclin D1 $A 870 G$ polymorphism in an Indian population. $J$ Cancer Res Clin Oncol, 132, 193-9.

Kristt D, Turner I, Koren R, et al (2000). Over expression of cyclin D1 mRNA in colorectal carcinomas and relationship to clinicopathological features: an in situ hybridization analysis. Pathol Oncol Res, 6, 65-70.

Li Y, Wei J, Xu C, et al (2014). Prognostic significance of cyclin DI expression in colorectal cancer: a meta-analysis of observational studies. PLoS one, 9, e94508.

Maeda K, Chung YS, Kang SM, et al (1997). Overexpression of cyclin D1 and $p 53$ associated with disease recurrence in colorectal adenocarcinoma. Int $J$ Cancer, 74, 310-5.

Mate JL, Ariza A, Aracil C, et al (1996). Cyclin D1 overexpression in non-small cell lung carcinoma: correlation with $\mathrm{Ki}-67$ labeling index and poor cytoplasmic differentiation. $J$ Pathol, 180, 395-9.

McKay JA, Douglas JJ, Ross VG, et al (2002). Analysis of key cell-cycle checkpoint proteins in colorectal tumours. J Pathol, 196, 386-93.

Mermelshtein A, Gerson A, Walfisch S, et al (2005). Expression of D-type cyclins in colon cancer and in cell lines from colon carcinomas. Br J Cancer, 93, 338-45.

Myklebust M, Li Z, Tran TH, et al (2012). Expression of cyclin $D 1 a$ and $D 1 b$ as predictive factors for treatmentresponse in colorectal cancer. Br J Cancer, 107, 1684-91.

Ogino S, Nosho K, Irahara N, et al (2009). A cohortstudy of cyclin D1 expression and prognosis in 602 colon cancercases. Clin Cancer Res, 15, 4431-8.

Pasz-Walczak G, Kordek R, Faflik M (2001). P21 (WAF1) expression in colorectal cancer: correlation with P53 and cyclin D1 expression, clinicopathological parameters and prognosis. Pathol Res Pract, 197, 683-9.

Sterlacci W, Fiegl M, Hilbe W, et al (2010). Deregulation of p27 and cyclin D1/D3 control over mitosis is associated with unfavorable prognosis in non-small cell lung cancer, as determined in 405 operated patients. $J$ Thorac Oncol, 5, 1325-36.

Tashiro E, Tsuchiya A, Imoto M (2007). Functions of cyclin $D 1$ as an oncogene and regulation of cyclin D1 expression. Cancer Sci, 98, 629-35.

Von Stockmar-Von Wangenheim CA, Monig SP, Schneider PM, et al (2008). P16, cyclin D1 and $R b$ expression in colorectalcarcinomas: Correlations with clinico-pathological parameters and prognosis. Mol Med Rep, 1, 27-32.

Xu XL, Chen SZ, Chen W, et al (2013). The impact of cyclin $D 1$ overexpression on the prognosis of ER-positive breast cancers: a metaanalysis. Breast Cancer Res Treat, 139, 329-39.

Zhao J, Li L, Wei S, et al (2012). Clinicopathological and prognostic role of cyclin D1 in esophageal squamous cell carcinoma: a meta-analysis. Dis Esophagus, 25, 520-6.

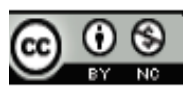

This work is licensed under a Creative Commons AttributionNon Commercial 4.0 International License. 